\title{
Spitzer/MIPS Imaging of the Extremely Extended Dust Shell(s) around R Hya
}

\author{
Angela K. Speck ${ }^{1}$, Toshiya Ueta ${ }^{2}$ and the MIRIAD team ${ }^{3}$ \\ ${ }^{1}$ Physics \& Astronomy, University of Missouri, Columbia, MO 65211, USA \\ email: speckan@missouri.edu \\ ${ }^{2}$ NASA Ames Research Center/USRA SOFIA Office, Mail Stop 211-3, Moffett Field, \\ CA 94035.
}

${ }^{3}$ R. Stencel (University of Denver), R. Gehrz (University of Minnesota), W. Latter (IPAC), M. Meixner (STScI), M. Elitzur (University of Kentucky), F. Herwig (LANL), H. Izumiura (NAOJ), M. Matsuura (Queen's University, Belfast), R. Szczerba (NCAC), M. Steffen (AIP), A. Zijlstra (University of Manchester)

Abstract. We present Spitzer/MIPS far-infrared (FIR) mapping of O-rich AGB star, R Hya.

Keywords. stars: AGB and post-AGB, stars: circumstellar matter, stars: mass loss

\section{Introduction}

The progenitors of planetary nebulae $(\mathrm{PNe})$ are stars with initial masses in the 0.8 $8 \mathrm{M}_{\odot}$ range. These stars eventually evolve into asymptotic giant branch (AGB) stars, at which time they begin to lose much of their mass, leading to the formation of a circumstellar shell of gas and dust. At the end of the AGB phase, mass loss stops and the circumstellar shell begins to drift away from the star. As the circumstellar shell disperses into the interstellar medium (ISM) these stars move to the blue side of the H-R diagram, evolving towards the PN phase. Assuming outflow velocities remain constant during the AGB phase, circumstellar shells of AGB and post-AGB stars contain the fossil record of their mass loss, and therefore have the potential to verify many aspects of stellar evolution.

IRAS and ISO data indicate that huge dust shells exist around many such objects, extending several parsecs from the central star (Young, Phillips \& Knapp 1993). Furthermore, some of these large dust shells show evidence for mass-loss variations that correlate with evolutionary changes in the star itself (Speck, Meixner \& Knapp 2000). Previous observations lacked the sensitivity and spatial resolution to investigate the full extent and detailed structure of these large dust shells.

Using Spitzer/MIPS's unique sensitivity and mapping capabilities, we are mapping a sample of very extended dust shells around (post-)AGB stars. From this study we will be able to (a) constrain the masses of the progenitor stars; (b) test theories of stellar evolution and mass-loss mechanisms; (c) determine the effect of dust chemistry on mass loss (and therefore on stellar evolution); and (d) determine when the aspherical structures so prevalent in planetary nebulae actually occur and thus constrain the cause.

Here we present Spitzer/MIPS far-infrared (FIR) maps of the oxygen-rich AGB star R Hya.

\section{Observations}

Figure 1 shows the Spitzer MIPS images of R Hya at 70 (top) and 160 $\mu \mathrm{m}$ (bottom). At $160 \mu \mathrm{m}, \mathrm{R}$ Hya exhibits a spiral arm structure, extended out to $\sim 300^{\prime \prime}$. The $70 \mu \mathrm{m}$ 


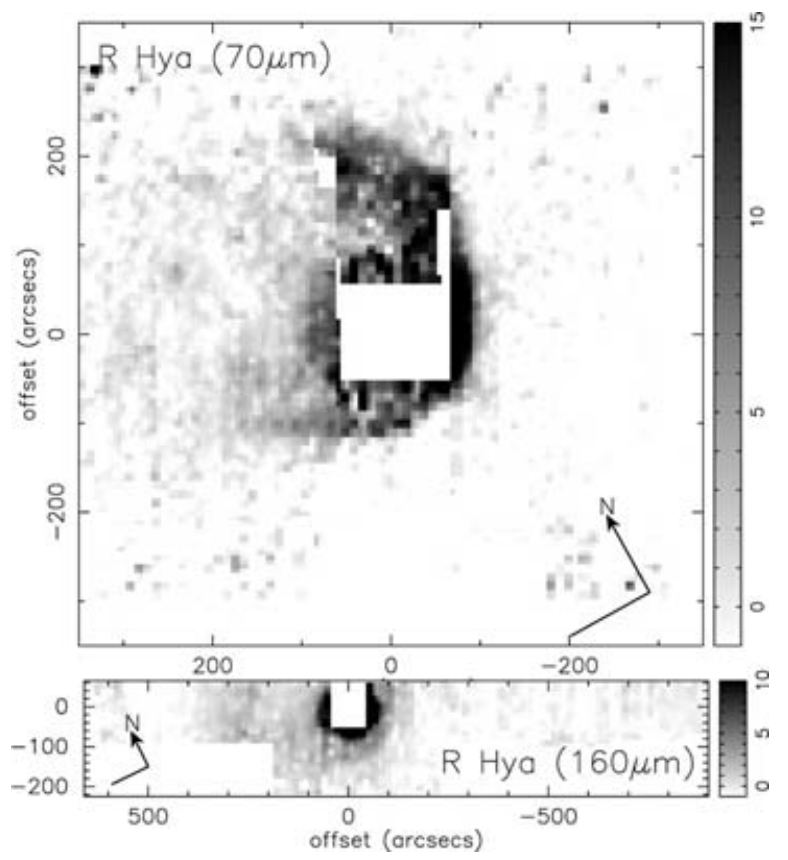

Figure 1. Spitzer/MIPS images of R Hya. Top: $70 \mu \mathrm{m}$. Bottom: $160 \mu \mathrm{m}$. Greyscale is surface brightness in MJy/sr

image of R Hya shows a similar spiral structure, but with a different orientation. The extended spiral arm seen at $160 \mu \mathrm{m}$ is located in the gap in the outer shell seen at $70 \mu \mathrm{m}$. The $160 \mu \mathrm{m}$ observations are more sensitive, implying that the upper left region of the outer shell seen in Figure $1 a$ is either cooler or contains less dust (or both).

The extended emission has been seen previously in IRAS images (Hashimoto et al. 1998), and was interpreted to be a detached dust shell. Comparison of the MIPS brightness profiles at $70 \mu \mathrm{m}$ and $160 \mu \mathrm{m}$ for $\mathrm{R}$ Hya shows that there is a double shell structure. This may imply the the previous IRAS images could not resolve the inner dust, although its presence was hypothesized in order to match the $25 \mu \mathrm{m}$ brightness.

The shape of the dust emission suggests a bow shock structure where the circumstellar shell interacts with the ISM (Wareing et al. 2006). The directionality of this bow shock is consistent with the star's proper motion (Eggen 1985).

\section{Conclusions}

R Hya exibits an extended double shell structure. Furthermore, this object shows evidence of bow shock interaction with the ISM. The shape of the bow shock is consistent with the star's direction of proper motion.

\section{References}

Eggen, O.J. 1985, AJ 90, 74.

Hashimoto, O., Izumiura, H., Kester, D. J. M., \& Bontekoe, Tj. R. 1998, A\&A 329, 213.

Speck, A.K., Meixner, M., \& Knapp, G.R. 2000, ApJL 545, L145.

Wareing, C. et al. 2006, MNRAS 366, 387.

Young, K., Phillips, T.G., \& Knapp, G.R. 1993 ApJ 409, 725. 